\title{
A large annotated corpus for learning natural language inference
}

\author{
Samuel R. Bowman*† \\ sbowmanestanford.edu \\ Christopher Potts* \\ cgpotts@stanford.edu
}

\author{
Gabor Angeli ${ }^{\dagger \ddagger}$ \\ angeli@stanford.edu
}

\author{
Christopher D. Manning ${ }^{* \dagger \ddagger}$ \\ manning@stanford.edu
}

${ }^{*}$ Stanford Linguistics $\quad{ }^{\dagger}$ Stanford NLP Group $\quad{ }^{\ddagger}$ Stanford Computer Science

\begin{abstract}
Understanding entailment and contradiction is fundamental to understanding natural language, and inference about entailment and contradiction is a valuable testing ground for the development of semantic representations. However, machine learning research in this area has been dramatically limited by the lack of large-scale resources. To address this, we introduce the Stanford Natural Language Inference corpus, a new, freely available collection of labeled sentence pairs, written by humans doing a novel grounded task based on image captioning. At $570 \mathrm{~K}$ pairs, it is two orders of magnitude larger than all other resources of its type. This increase in scale allows lexicalized classifiers to outperform some sophisticated existing entailment models, and it allows a neural network-based model to perform competitively on natural language inference benchmarks for the first time.
\end{abstract}

\section{Introduction}

The semantic concepts of entailment and contradiction are central to all aspects of natural language meaning (Katz, 1972; van Benthem, 2008), from the lexicon to the content of entire texts. Thus, natural language inference (NLI) - characterizing and using these relations in computational systems (Fyodorov et al., 2000; Condoravdi et al., 2003; Bos and Markert, 2005; Dagan et al., 2006; MacCartney and Manning, 2009) — is essential in tasks ranging from information retrieval to semantic parsing to commonsense reasoning.

NLI has been addressed using a variety of techniques, including those based on symbolic logic, knowledge bases, and neural networks. In recent years, it has become an important testing ground for approaches employing distributed word and phrase representations. Distributed representations excel at capturing relations based in similarity, and have proven effective at modeling simple dimensions of meaning like evaluative sentiment (e.g., Socher et al. 2013), but it is less clear that they can be trained to support the full range of logical and commonsense inferences required for NLI (Bowman et al., 2015; Weston et al., 2015b; Weston et al., 2015a). In a SemEval 2014 task aimed at evaluating distributed representations for $\mathrm{NLI}$, the best-performing systems relied heavily on additional features and reasoning capabilities (Marelli et al., 2014a).

Our ultimate objective is to provide an empirical evaluation of learning-centered approaches to NLI, advancing the case for NLI as a tool for the evaluation of domain-general approaches to semantic representation. However, in our view, existing NLI corpora do not permit such an assessment. They are generally too small for training modern data-intensive, wide-coverage models, many contain sentences that were algorithmically generated, and they are often beset with indeterminacies of event and entity coreference that significantly impact annotation quality.

To address this, this paper introduces the Stanford Natural Language Inference (SNLI) corpus, a collection of sentence pairs labeled for entailment, contradiction, and semantic independence. At 570,152 sentence pairs, SNLI is two orders of magnitude larger than all other resources of its type. And, in contrast to many such resources, all of its sentences and labels were written by humans in a grounded, naturalistic context. In a separate validation phase, we collected four additional judgments for each label for 56,941 of the examples. Of these, $98 \%$ of cases emerge with a threeannotator consensus, and 58\% see a unanimous consensus from all five annotators.

In this paper, we use this corpus to evaluate 


\begin{tabular}{|c|c|c|}
\hline $\begin{array}{l}\text { A man inspects the uniform of a figure in some East } \\
\text { Asian country. }\end{array}$ & $\begin{array}{l}\text { contradiction } \\
\text { C C C C C }\end{array}$ & The man is sleeping \\
\hline An older and younger man smiling. & $\begin{array}{l}\text { neutral } \\
\text { N N E N N }\end{array}$ & $\begin{array}{l}\text { Two men are smiling and laughing at the cats play- } \\
\text { ing on the floor. }\end{array}$ \\
\hline $\begin{array}{l}\text { A black race car starts up in front of a crowd of } \\
\text { people. }\end{array}$ & $\begin{array}{l}\text { contradiction } \\
\text { C C C C C }\end{array}$ & A man is driving down a lonely road. \\
\hline A soccer game with multiple males playing. & $\begin{array}{l}\text { entailment } \\
\text { E E E E E }\end{array}$ & Some men are playing a sport. \\
\hline $\begin{array}{l}\text { A smiling costumed woman is holding an um- } \\
\text { brella. }\end{array}$ & $\begin{array}{l}\text { neutral } \\
\text { N N E C N }\end{array}$ & $\begin{array}{l}\text { A happy woman in a fairy costume holds an um- } \\
\text { brella. }\end{array}$ \\
\hline
\end{tabular}

Table 1: Randomly chosen examples from the development section of our new corpus, shown with both the selected gold labels and the full set of labels (abbreviated) from the individual annotators, including (in the first position) the label used by the initial author of the pair.

a variety of models for natural language inference, including rule-based systems, simple linear classifiers, and neural network-based models. We find that two models achieve comparable performance: a feature-rich classifier model and a neural network model centered around a Long Short-Term Memory network (LSTM; Hochreiter and Schmidhuber 1997). We further evaluate the LSTM model by taking advantage of its ready support for transfer learning, and show that it can be adapted to an existing NLI challenge task, yielding the best reported performance by a neural network model and approaching the overall state of the art.

\section{A new corpus for NLI}

To date, the primary sources of annotated NLI corpora have been the Recognizing Textual Entailment (RTE) challenge tasks. ${ }^{1}$ These are generally high-quality, hand-labeled data sets, and they have stimulated innovative logical and statistical models of natural language reasoning, but their small size (fewer than a thousand examples each) limits their utility as a testbed for learned distributed representations. The data for the SemEval 2014 task called Sentences Involving Compositional Knowledge (SICK) is a step up in terms of size, but only to 4,500 training examples, and its partly automatic construction introduced some spurious patterns into the data (Marelli et al. 2014a, §6). The Denotation Graph entailment set (Young et al., 2014) contains millions of examples of entailments between sentences and artificially constructed short phrases, but it was labeled using fully automatic methods, and is noisy enough that it is probably suitable only as a source of sup-

\footnotetext{
${ }^{1}$ http://aclweb. org/aclwiki/index.php? title=Textual_Entailment_Resource_Pool
}

plementary training data. Outside the domain of sentence-level entailment, Levy et al. (2014) introduce a large corpus of semi-automatically annotated entailment examples between subject-verbobject relation triples, and the second release of the Paraphrase Database (Pavlick et al., 2015) includes automatically generated entailment annotations over a large corpus of pairs of words and short phrases.

Existing resources suffer from a subtler issue that impacts even projects using only humanprovided annotations: indeterminacies of event and entity coreference lead to insurmountable indeterminacy concerning the correct semantic label (de Marneffe et al. $2008 \S 4.3$; Marelli et al. 2014b). For an example of the pitfalls surrounding entity coreference, consider the sentence pair A boat sank in the Pacific Ocean and A boat sank in the Atlantic Ocean. The pair could be labeled as a contradiction if one assumes that the two sentences refer to the same single event, but could also be reasonably labeled as neutral if that assumption is not made. In order to ensure that our labeling scheme assigns a single correct label to every pair, we must select one of these approaches across the board, but both choices present problems. If we opt not to assume that events are coreferent, then we will only ever find contradictions between sentences that make broad universal assertions, but if we opt to assume coreference, new counterintuitive predictions emerge. For example, Ruth Bader Ginsburg was appointed to the US Supreme Court and I had a sandwich for lunch today would unintuitively be labeled as a contradiction, rather than neutral, under this assumption.

Entity coreference presents a similar kind of indeterminacy, as in the pair A tourist visited New 
York and A tourist visited the city. Assuming coreference between New York and the city justifies labeling the pair as an entailment, but without that assumption the city could be taken to refer to a specific unknown city, leaving the pair neutral. This kind of indeterminacy of label can be resolved only once the questions of coreference are resolved.

With SNLI, we sought to address the issues of size, quality, and indeterminacy. To do this, we employed a crowdsourcing framework with the following crucial innovations. First, the examples were grounded in specific scenarios, and the premise and hypothesis sentences in each example were constrained to describe that scenario from the same perspective, which helps greatly in controlling event and entity coreference. ${ }^{2}$ Second, the prompt gave participants the freedom to produce entirely novel sentences within the task setting, which led to richer examples than we see with the more proscribed string-editing techniques of earlier approaches, without sacrificing consistency. Third, a subset of the resulting sentences were sent to a validation task aimed at providing a highly reliable set of annotations over the same data, and at identifying areas of inferential uncertainty.

\subsection{Data collection}

We used Amazon Mechanical Turk for data collection. In each individual task (each HIT), a worker was presented with premise scene descriptions from a pre-existing corpus, and asked to supply hypotheses for each of our three labelsentailment, neutral, and contradiction-forcing the data to be balanced among these classes.

The instructions that we provided to the workers are shown in Figure 1. Below the instructions were three fields for each of three requested sentences, corresponding to our entailment, neutral, and contradiction labels, a fourth field (marked optional) for reporting problems, and a link to an FAQ page. That FAQ grew over the course of data collection. It warned about disallowed techniques (e.g., reusing the same sentence for many different prompts, which we saw in a few cases), provided guidance concerning sentence length and

\footnotetext{
2 Issues of coreference are not completely solved, bu greatly mitigated. For example, with the premise sentence A dog is lying in the grass, a worker could safely assume that the dog is the most prominent thing in the photo, and very likely the only dog, and build contradicting sentences assuming reference to the same dog.
}

We will show you the caption for a photo. We will not show you the photo. Using only the caption and what you know about the world:

- Write one alternate caption that is definitely a true description of the photo. Example: For the caption "Two dogs are running through a field." you could write "There are animals outdoors."

- Write one alternate caption that might be a true description of the photo. Example: For the caption "Two dogs are running through a field." you could write "Some puppies are running to catch a stick."

- Write one alternate caption that is definitely a false description of the photo. Example: For the caption "Two dogs are running through a field." you could write "The pets are sitting on a couch." This is different from the maybe correct category because it's impossible for the dogs to be both running and sitting.

Figure 1: The instructions used on Mechanical Turk for data collection.

complexity (we did not enforce a minimum length, and we allowed bare NPs as well as full sentences), and reviewed logistical issues around payment timing. About 2,500 workers contributed.

For the premises, we used captions from the Flickr30k corpus (Young et al., 2014), a collection of approximately $160 \mathrm{k}$ captions (corresponding to about 30k images) collected in an earlier crowdsourced effort. ${ }^{3}$ The captions were not authored by the photographers who took the source images, and they tend to contain relatively literal scene descriptions that are suited to our approach, rather than those typically associated with personal photographs (as in their example: Our trip to the Olympic Peninsula). In order to ensure that the label for each sentence pair can be recovered solely based on the available text, we did not use the images at all during corpus collection.

Table 2 reports some key statistics about the collected corpus, and Figure 2 shows the distributions of sentence lengths for both our source hypotheses and our newly collected premises. We observed that while premise sentences varied considerably in length, hypothesis sentences tended to be as

\footnotetext{
${ }^{3}$ We additionally include about $4 \mathrm{k}$ sentence pairs from a pilot study in which the premise sentences were instead drawn from the VisualGenome corpus (under construction; visualgenome.org). These examples appear only in the training set, and have pair identifiers prefixed with $\mathrm{vg}$ in our corpus.
} 


\begin{tabular}{lr}
\hline Data set sizes: & \\
Training pairs & 550,152 \\
Development pairs & 10,000 \\
Test pairs & 10,000 \\
\hline Sentence length: & \\
Premise mean token count & 14.1 \\
Hypothesis mean token count & 8.3 \\
\hline Parser output: & \\
Premise 'S'-rooted parses & $74.0 \%$ \\
Hypothesis 'S'-rooted parses & $88.9 \%$ \\
Distinct words (ignoring case) & 37,026 \\
\hline
\end{tabular}

Table 2: Key statistics for the raw sentence pairs in SNLI. Since the two halves of each pair were collected separately, we report some statistics for both.

short as possible while still providing enough information to yield a clear judgment, clustering at around seven words. We also observed that the bulk of the sentences from both sources were syntactically complete rather than fragments, and the frequency with which the parser produces a parse rooted with an ' $S$ ' (sentence) node attests to this.

\subsection{Data validation}

In order to measure the quality of our corpus, and in order to construct maximally useful testing and development sets, we performed an additional round of validation for about $10 \%$ of our data. This validation phase followed the same basic form as the Mechanical Turk labeling task used to label the SICK entailment data: we presented workers with pairs of sentences in batches of five, and asked them to choose a single label for each pair. We supplied each pair to four annotators, yielding five labels per pair including the label used by the original author. The instructions were similar to the instructions for initial data collection shown in Figure 1, and linked to a similar FAQ. Though we initially used a very restrictive qualification (based on past approval rate) to select workers for the validation task, we nonetheless discovered (and deleted) some instances of random guessing in an early batch of work, and subsequently instituted a fully closed qualification restricted to about 30 trusted workers.

For each pair that we validated, we assigned a gold label. If any one of the three labels was chosen by at least three of the five annotators, it was

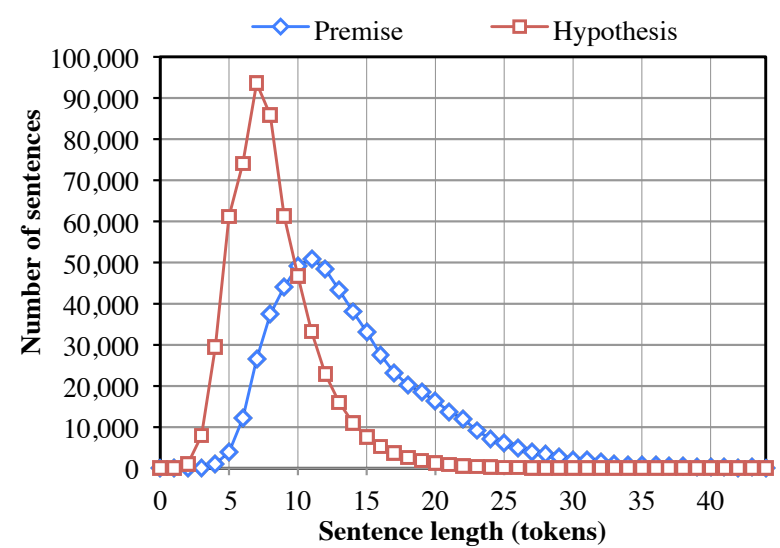

Figure 2: The distribution of sentence length.

chosen as the gold label. If there was no such consensus, which occurred in about $2 \%$ of cases, we assigned the placeholder label '-'. While these unlabeled examples are included in the corpus distribution, they are unlikely to be helpful for the standard NLI classification task, and we do not include them in either training or evaluation in the experiments that we discuss in this paper.

The results of this validation process are summarized in Table 3. Nearly all of the examples received a majority label, indicating broad consensus about the nature of the data and categories. The gold-labeled examples are very nearly evenly distributed across the three labels. The Fleiss $\kappa$ scores (computed over every example with a full five annotations) are likely to be conservative given our large and unevenly distributed pool of annotators, but they still provide insights about the levels of disagreement across the three semantic classes. This disagreement likely reflects not just the limitations of large crowdsourcing efforts but also the uncertainty inherent in naturalistic NLI. Regardless, the overall rate of agreement is extremely high, suggesting that the corpus is sufficiently high quality to pose a challenging but realistic machine learning task.

\subsection{The distributed corpus}

Table 1 shows a set of randomly chosen validated examples from the development set with their labels. Qualitatively, we find the data that we collected draws fairly extensively on commonsense knowledge, and that hypothesis and premise sentences often differ structurally in significant ways, suggesting that there is room for improvement beyond superficial word alignment models. We also find the sentences that we collected to be largely 


\begin{tabular}{|c|c|}
\hline \multicolumn{2}{|l|}{ General: } \\
\hline Validated pairs & 56,951 \\
\hline Pairs w/ unanimous gold label & $58.3 \%$ \\
\hline \multicolumn{2}{|c|}{ Individual annotator label agreement: } \\
\hline Individual label $=$ gold label & $89.0 \%$ \\
\hline Individual label $=$ author's label & $85.8 \%$ \\
\hline \multicolumn{2}{|c|}{ Gold label/author's label agreement: } \\
\hline Gold label = author's label & $91.2 \%$ \\
\hline Gold label $\neq$ author's label & $6.8 \%$ \\
\hline No gold label (no 3 labels match) & $2.0 \%$ \\
\hline \multicolumn{2}{|l|}{ Fleiss $\kappa$ : } \\
\hline contradiction & 0.77 \\
\hline entailment & 0.72 \\
\hline neutral & 0.60 \\
\hline Overall & 0.70 \\
\hline
\end{tabular}

Table 3: Statistics for the validated pairs. The $a u$ thor's label is the label used by the worker who wrote the premise to create the sentence pair. A gold label reflects a consensus of three votes from among the author and the four annotators.

fluent, correctly spelled English, with a mix of full sentences and caption-style noun phrase fragments, though punctuation and capitalization are often omitted.

The corpus is available under a CreativeCommons Attribution-ShareAlike license, the same license used for the Flickr30k source captions. It can be downloaded at:

nlp.stanford.edu/projects/snli/

Partition We distribute the corpus with a prespecified train/test/development split. The test and development sets contain 10k examples each. Each original ImageFlickr caption occurs in only one of the three sets, and all of the examples in the test and development sets have been validated.

Parses The distributed corpus includes parses produced by the Stanford PCFG Parser 3.5.2 (Klein and Manning, 2003), trained on the standard training set as well as on the Brown Corpus (Francis and Kucera 1979), which we found to improve the parse quality of the descriptive sentences and noun phrases found in the descriptions.

\section{Our data as a platform for evaluation}

The most immediate application for our corpus is in developing models for the task of NLI. In par-

\begin{tabular}{lccc}
\hline System & SNLI & SICK & RTE-3 \\
\hline Edit Distance Based & 71.9 & 65.4 & 61.9 \\
Classifier Based & 72.2 & 71.4 & 61.5 \\
$\quad$ + Lexical Resources & $\mathbf{7 5 . 0}$ & $\mathbf{7 8 . 8}$ & $\mathbf{6 3 . 6}$ \\
\hline
\end{tabular}

Table 4: 2-class test accuracy for two simple baseline systems included in the Excitement Open Platform, as well as SICK and RTE results for a model making use of more sophisticated lexical resources.

ticular, since it is dramatically larger than any existing corpus of comparable quality, we expect it to be suitable for training parameter-rich models like neural networks, which have not previously been competitive at this task. Our ability to evaluate standard classifier-base NLI models, however, was limited to those which were designed to scale to SNLI's size without modification, so a more complete comparison of approaches will have to wait for future work. In this section, we explore the performance of three classes of models which could scale readily: (i) models from a well-known NLI system, the Excitement Open Platform; (ii) variants of a strong but simple feature-based classifier model, which makes use of both unlexicalized and lexicalized features, and (iii) distributed representation models, including a baseline model and neural network sequence models.

\subsection{Excitement Open Platform models}

The first class of models is from the Excitement Open Platform (EOP, Padó et al. 2014; Magnini et al. 2014) - an open source platform for RTE research. EOP is a tool for quickly developing NLI systems while sharing components such as common lexical resources and evaluation sets. We evaluate on two algorithms included in the distribution: a simple edit-distance based algorithm and a classifier-based algorithm, the latter both in a bare form and augmented with EOP's full suite of lexical resources.

Our initial goal was to better understand the difficulty of the task of classifying SNLI corpus inferences, rather than necessarily the performance of a state-of-the-art RTE system. We approached this by running the same system on several data sets: our own test set, the SICK test data, and the standard RTE-3 test set (Giampiccolo et al., 2007). We report results in Table 4. Each of the models 
was separately trained on the training set of each corpus. All models are evaluated only on 2-class entailment. To convert 3-class problems like SICK and SNLI to this setting, all instances of contradiction and unknown are converted to nonentailment. This yields a most-frequent-class baseline accuracy of $66 \%$ on SNLI, and $71 \%$ on SICK. This is intended primarily to demonstrate the difficulty of the task, rather than necessarily the performance of a state-of-the-art RTE system. The edit distance algorithm tunes the weight of the three caseinsensitive edit distance operations on the training set, after removing stop words. In addition to the base classifier-based system distributed with the platform, we train a variant which includes information from WordNet (Miller, 1995) and VerbOcean (Chklovski and Pantel, 2004), and makes use of features based on tree patterns and dependency tree skeletons (Wang and Neumann, 2007).

\subsection{Lexicalized Classifier}

Unlike the RTE datasets, SNLI's size supports approaches which make use of rich lexicalized features. We evaluate a simple lexicalized classifier to explore the ability of non-specialized models to exploit these features in lieu of more involved language understanding. Our classifier implements 6 feature types; 3 unlexicalized and 3 lexicalized:

1. The BLEU score of the hypothesis with respect to the premise, using an n-gram length between 1 and 4 .

2. The length difference between the hypothesis and the premise, as a real-valued feature.

3. The overlap between words in the premise and hypothesis, both as an absolute count and a percentage of possible overlap, and both over all words and over just nouns, verbs, adjectives, and adverbs.

4. An indicator for every unigram and bigram in the hypothesis.

5. Cross-unigrams: for every pair of words across the premise and hypothesis which share a POS tag, an indicator feature over the two words.

6. Cross-bigrams: for every pair of bigrams across the premise and hypothesis which share a POS tag on the second word, an indicator feature over the two bigrams.

We report results in Table 5, along with ablation studies for removing the cross-bigram features (leaving only the cross-unigram feature) and

\begin{tabular}{lcccc}
\hline \multirow{2}{*}{ System } & \multicolumn{2}{c}{ SNLI } & \multicolumn{2}{c}{ SICK } \\
& Train & Test & Train & Test \\
\hline Lexicalized & 99.7 & $\mathbf{7 8 . 2}$ & 90.4 & $\mathbf{7 7 . 8}$ \\
Unigrams Only & 93.1 & 71.6 & 88.1 & 77.0 \\
Unlexicalized & 49.4 & 50.4 & 69.9 & 69.6 \\
\hline
\end{tabular}

Table 5: 3-class accuracy, training on either our data or SICK, including models lacking crossbigram features (Feature 6), and lacking all lexical features (Features 4-6). We report results both on the test set and the training set to judge overfitting.

for removing all lexicalized features. On our large corpus in particular, there is a substantial jump in accuracy from using lexicalized features, and another from using the very sparse cross-bigram features. The latter result suggests that there is value in letting the classifier automatically learn to recognize structures like explicit negations and adjective modification. A similar result was shown in Wang and Manning (2012) for bigram features in sentiment analysis.

It is surprising that the classifier performs as well as it does without any notion of alignment or tree transformations. Although we expect that richer models would perform better, the results suggest that given enough data, cross bigrams with the noisy part-of-speech overlap constraint can produce an effective model.

\subsection{Sentence embeddings and NLI}

SNLI is suitably large and diverse to make it possible to train neural network models that produce distributed representations of sentence meaning. In this section, we compare the performance of three such models on the corpus. To focus specifically on the strengths of these models at producing informative sentence representations, we use sentence embedding as an intermediate step in the NLI classification task: each model must produce a vector representation of each of the two sentences without using any context from the other sentence, and the two resulting vectors are then passed to a neural network classifier which predicts the label for the pair. This choice allows us to focus on existing models for sentence embedding, and it allows us to evaluate the ability of those models to learn useful representations of meaning (which may be independently useful for subsequent tasks), at the cost of excluding from con- 


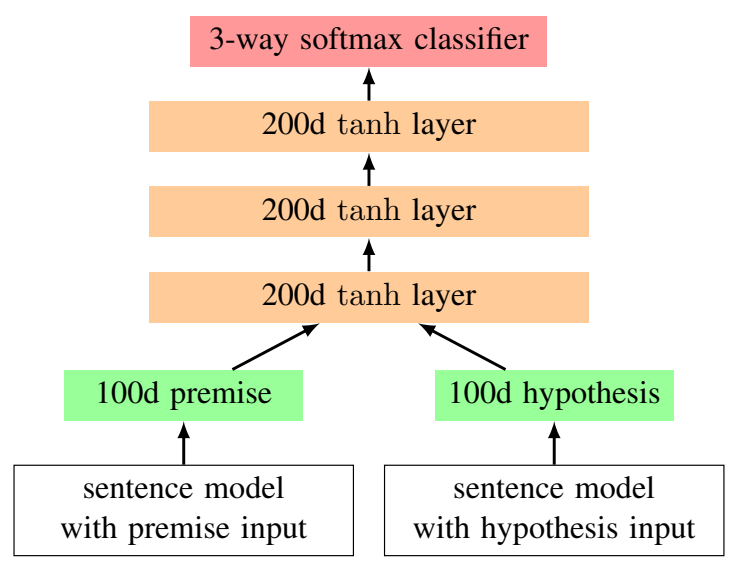

Figure 3: The neural network classification architecture: for each sentence embedding model evaluated in Tables 6 and 7, two identical copies of the model are run with the two sentences as input, and their outputs are used as the two 100d inputs shown here.

sideration possible strong neural models for NLI that directly compare the two inputs at the word or phrase level.

Our neural network classifier, depicted in Figure 3 (and based on a one-layer model in Bowman et al. 2015), is simply a stack of three $200 \mathrm{~d}$ tanh layers, with the bottom layer taking the concatenated sentence representations as input and the top layer feeding a softmax classifier, all trained jointly with the sentence embedding model itself.

We test three sentence embedding models, each set to use 100d phrase and sentence embeddings. Our baseline sentence embedding model simply sums the embeddings of the words in each sentence. In addition, we experiment with two simple sequence embedding models: a plain RNN and an LSTM RNN (Hochreiter and Schmidhuber, 1997).

The word embeddings for all of the models are initialized with the $300 \mathrm{~d}$ reference GloVe vectors (840B token version, Pennington et al. 2014) and fine-tuned as part of training. In addition, all of the models use an additional tanh neural network layer to map these $300 \mathrm{~d}$ embeddings into the lower-dimensional phrase and sentence embedding space. All of the models are randomly initialized using standard techniques and trained using AdaDelta (Zeiler, 2012) minibatch SGD until performance on the development set stops improving. We applied L2 regularization to all models, manually tuning the strength coefficient $\lambda$ for each, and additionally applied dropout (Srivastava et al., 2014) to the inputs and outputs of the sen-

\begin{tabular}{lcc}
\hline Sentence model & Train & Test \\
\hline 100d Sum of words & 79.3 & 75.3 \\
100d RNN & 73.1 & 72.2 \\
100d LSTM RNN & 84.8 & $\mathbf{7 7 . 6}$ \\
\hline
\end{tabular}

Table 6: Accuracy in 3-class classification on our training and test sets for each model.

tence embedding models (though not to its internal connections) with a fixed dropout rate. All models were implemented in a common framework for this paper, and the implementations will be made available at publication time.

The results are shown in Table 6. The sum of words model performed slightly worse than the fundamentally similar lexicalized classifierwhile the sum of words model can use pretrained word embeddings to better handle rare words, it lacks even the rudimentary sensitivity to word order that the lexicalized model's bigram features provide. Of the two RNN models, the LSTM's more robust ability to learn long-term dependencies serves it well, giving it a substantial advantage over the plain RNN, and resulting in performance that is essentially equivalent to the lexicalized classifier on the test set (LSTM performance near the stopping iteration varies by up to $0.5 \%$ between evaluation steps). While the lexicalized model fits the training set almost perfectly, the gap between train and test set accuracy is relatively small for all three neural network models, suggesting that research into significantly higher capacity versions of these models would be productive.

\subsection{Analysis and discussion}

Figure 4 shows a learning curve for the LSTM and the lexicalized and unlexicalized feature-based models. It shows that the large size of the corpus is crucial to both the LSTM and the lexicalized model, and suggests that additional data would yield still better performance for both. In addition, though the LSTM and the lexicalized model show similar performance when trained on the current full corpus, the somewhat steeper slope for the LSTM hints that its ability to learn arbitrarily structured representations of sentence meaning may give it an advantage over the more constrained lexicalized model on still larger datasets.

We were struck by the speed with which the lexicalized classifier outperforms its unlexicalized 


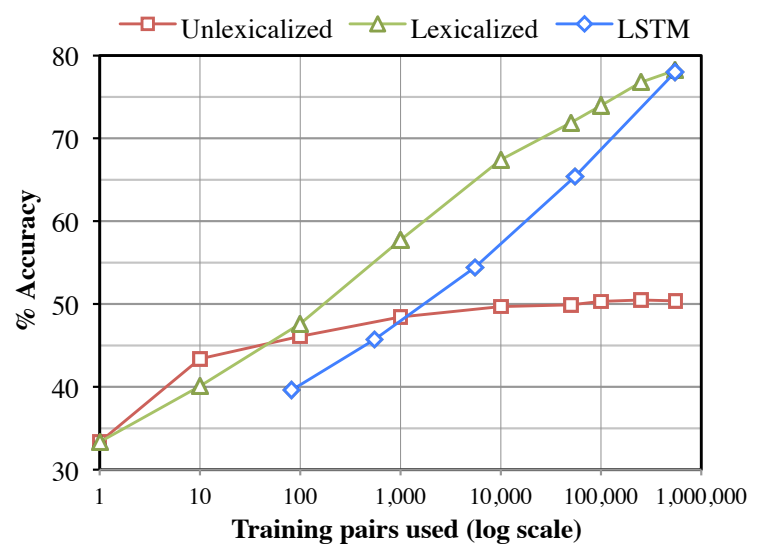

Figure 4: A learning curve showing how the baseline classifiers and the LSTM perform when trained to convergence on varied amounts of training data. The $y$-axis starts near a random-chance accuracy of $33 \%$. The minibatch size of 64 that we used to tune the LSTM sets a lower bound on data for that model.

counterpart. With only 100 training examples, the cross-bigram classifier is already performing better. Empirically, we find that the top weighted features for the classifier trained on 100 examples tend to be high precision entailments; e.g., playing $\rightarrow$ outside (most scenes are outdoors), a banana $\rightarrow$ person eating. If relatively few spurious entailments get high weight - as it appears is the casethen it makes sense that, when these do fire, they boost accuracy in identifying entailments.

There are revealing patterns in the errors common to all the models considered here. Despite the large size of the training corpus and the distributional information captured by GloVe initialization, many lexical relationships are still misanalyzed, leading to incorrect predictions of independent, even for pairs that are common in the training corpus like beach/surf and sprinter/runner. Semantic mistakes at the phrasal level (e.g., predicting contradiction for $A$ male is placing an order in a deli/A man buying a sandwich at a deli) indicate that additional attention to compositional semantics would pay off. However, many of the persistent problems run deeper, to inferences that depend on world knowledge and contextspecific inferences, as in the entailment pair $A$ race car driver leaps from a burning carlA race car driver escaping danger, for which both the lexicalized classifier and the LSTM predict neutral. In other cases, the models' attempts to shortcut this kind of inference through lexical cues can lead them astray. Some of these examples have qualities reminiscent of Winograd schemas (Winograd, 1972; Levesque, 2013). For example, all the models wrongly predict entailment for $A$ young girl throws sand toward the ocean/A girl can't stand the ocean, presumably because of distributional associations between throws and can't stand.

Analysis of the models' predictions also yields insights into the extent to which they grapple with event and entity coreference. For the most part, the original image prompts contained a focal element that the caption writer identified with a syntactic subject, following information structuring conventions associating subjects and topics in English (Ward and Birner, 2004). Our annotators generally followed suit, writing sentences that, while structurally diverse, share topic/focus (theme/rheme) structure with their premises. This promotes a coherent, situation-specific construal of each sentence pair. This is information that our models can easily take advantage of, but it can lead them astray. For instance, all of them stumble with the amusingly simple case $A$ woman prepares ingredients for a bowl of soup/A soup bowl prepares a woman, in which prior expectations about parallelism are not met. Another headline example of this type is A man wearing padded arm protection is being bitten by a German shepherd dog/A man bit a dog, which all the models wrongly diagnose as entailment, though the sentences report two very different stories. A model with access to explicit information about syntactic or semantic structure should perform better on cases like these.

\section{Transfer learning with SICK}

To the extent that successfully training a neural network model like our LSTM on SNLI forces that model to encode broadly accurate representations of English scene descriptions and to build an entailment classifier over those relations, we should expect it to be readily possible to adapt the trained model for use on other NLI tasks. In this section, we evaluate on the SICK entailment task using a simple transfer learning method (Pratt et al., 1991) and achieve competitive results.

To perform transfer, we take the parameters of the LSTM RNN model trained on SNLI and use them to initialize a new model, which is trained from that point only on the training portion of SICK. The only newly initialized parameters are 


\begin{tabular}{lrc}
\hline Training sets & Train & Test \\
\hline Our data only & 42.0 & 46.7 \\
SICK only & 100.0 & 71.3 \\
Our data and SICK (transfer) & 99.9 & $\mathbf{8 0 . 8}$ \\
\hline
\end{tabular}

Table 7: LSTM 3-class accuracy on the SICK train and test sets under three training regimes.

softmax layer parameters and the embeddings for words that appear in SICK, but not in SNLI (which are populated with GloVe embeddings as above). We use the same model hyperparameters that were used to train the original model, with the exception of the L2 regularization strength, which is re-tuned. We additionally transfer the accumulators that are used by AdaDelta to set the learning rates. This lowers the starting learning rates, and is intended to ensure that the model does not learn too quickly in its first few epochs after transfer and destroy the knowledge accumulated in the pre-transfer phase of training.

The results are shown in Table 7. Training on SICK alone yields poor performance, and the model trained on SNLI fails when tested on SICK data, labeling more neutral examples as contradictions than correctly, possibly as a result of subtle differences in how the labeling task was presented. In contrast, transferring SNLI representations to SICK yields the best performance yet reported for an unaugmented neural network model, surpasses the available EOP models, and approaches both the overall state of the art at $84.6 \%$ (Lai and Hockenmaier, 2014) and the $84 \%$ level of interannotator agreement, which likely represents an approximate performance ceiling. This suggests that the introduction of a large high-quality corpus makes it possible to train representation-learning models for sentence meaning that are competitive with the best hand-engineered models on inference tasks.

We attempted to apply this same transfer evaluation technique to the RTE-3 challenge, but found that the small training set (800 examples) did not allow the model to adapt to the unfamiliar genre of text used in that corpus, such that no training configuration yielded competitive performance. Further research on effective transfer learning on small data sets with neural models might facilitate improvements here.

\section{Conclusion}

Natural languages are powerful vehicles for reasoning, and nearly all questions about meaningfulness in language can be reduced to questions of entailment and contradiction in context. This suggests that NLI is an ideal testing ground for theories of semantic representation, and that training for NLI tasks can provide rich domain-general semantic representations. To date, however, it has not been possible to fully realize this potential due to the limited nature of existing NLI resources. This paper sought to remedy this with a new, largescale, naturalistic corpus of sentence pairs labeled for entailment, contradiction, and independence. We used this corpus to evaluate a range of models, and found that both simple lexicalized models and neural network models perform well, and that the representations learned by a neural network model on our corpus can be used to dramatically improve performance on a standard challenge dataset. We hope that SNLI presents valuable training data and a challenging testbed for the continued application of machine learning to semantic representation.

\section{Acknowledgments}

We gratefully acknowledge support from a Google Faculty Research Award, a gift from Bloomberg L.P., the Defense Advanced Research Projects Agency (DARPA) Deep Exploration and Filtering of Text (DEFT) Program under Air Force Research Laboratory (AFRL) contract no. FA875013-2-0040, the National Science Foundation under grant no. IIS 1159679, and the Department of the Navy, Office of Naval Research, under grant no. N00014-10-1-0109. Any opinions, findings, and conclusions or recommendations expressed in this material are those of the authors and do not necessarily reflect the views of Google, Bloomberg L.P., DARPA, AFRL NSF, ONR, or the US government. We also thank our many excellent Mechanical Turk contributors.

\section{References}

Johan Bos and Katja Markert. 2005. Recognising textual entailment with logical inference. In Proc. EMNLP.

Samuel R. Bowman, Christopher Potts, and Christopher D. Manning. 2015. Recursive neural networks can learn logical semantics. In Proc. of the 3rd Workshop on Continuous Vector Space Models and their Compositionality. 
Timothy Chklovski and Patrick Pantel. 2004. VerbOcean: Mining the web for fine-grained semantic verb relations. In Proc. EMNLP.

Cleo Condoravdi, Dick Crouch, Valeria de Paiva, Reinhard Stolle, and Daniel G. Bobrow. 2003. Entailment, intensionality and text understanding. In Proc. of the HLT-NAACL 2003 Workshop on Text Meaning.

Ido Dagan, Oren Glickman, and Bernardo Magnini. 2006. The PASCAL recognising textual entailment challenge. In Machine learning challenges. Evaluating predictive uncertainty, visual object classification, and recognising tectual entailment, pages 177190. Springer.

Marie-Catherine de Marneffe, Anna N. Rafferty, and Christopher D. Manning. 2008. Finding contradictions in text. In Proc. ACL.

W. Nelson Francis and Henry Kucera. 1979. Brown corpus manual. Brown University.

Yaroslav Fyodorov, Yoad Winter, and Nissim Francez. 2000. A natural logic inference system. In Proc. of the 2nd Workshop on Inference in Computational Semantics.

Danilo Giampiccolo, Bernardo Magnini, Ido Dagan, and Bill Dolan. 2007. The third PASCAL recognizing textual entailment challenge. In Proc. of the ACL-PASCAL workshop on textual entailment and paraphrasing.

Sepp Hochreiter and Jürgen Schmidhuber. 1997. Long short-term memory. Neural computation, 9(8):1735-1780.

Jerrold J. Katz. 1972. Semantic Theory. Harper \& Row, New York.

Dan Klein and Christopher D. Manning. 2003. Accurate unlexicalized parsing. In Proc. ACL.

Alice Lai and Julia Hockenmaier. 2014. Illinois-LH: A denotational and distributional approach to semantics. In Proc. SemEval.

Hector J. Levesque. 2013. On our best behaviour. In Proc. AAAI.

Omer Levy, Ido Dagan, and Jacob Goldberger. 2014. Focused entailment graphs for open IE propositions. In Proc. CoNLL.

Bill MacCartney and Christopher D Manning. 2009. An extended model of natural logic. In Proc. of the Eighth International Conference on Computational Semantics.

Bernardo Magnini, Roberto Zanoli, Ido Dagan, Kathrin Eichler, Günter Neumann, Tae-Gil Noh, Sebastian Pado, Asher Stern, and Omer Levy. 2014. The Excitement Open Platform for textual inferences. Proc. $A C L$.
Marco Marelli, Luisa Bentivogli, Marco Baroni, Raffaella Bernardi, Stefano Menini, and Roberto Zamparelli. 2014a. SemEval-2014 task 1: Evaluation of compositional distributional semantic models on full sentences through semantic relatedness and textual entailment. In Proc. SemEval.

Marco Marelli, Stefano Menini, Marco Baroni, Luisa Bentivogli, Raffaella Bernardi, and Roberto Zamparelli. 2014b. A SICK cure for the evaluation of compositional distributional semantic models. In Proc. LREC.

George A Miller. 1995. WordNet: a lexical database for english. Communications of the ACM, 38(11):39-41.

Sebastian Padó, Tae-Gil Noh, Asher Stern, Rui Wang, and Roberto Zanoli. 2014. Design and realization of a modular architecture for textual entailment. Journal of Natural Language Engineering.

Ellie Pavlick, Johan Bos, Malvina Nissim, Charley Beller, Ben Van Durme, and Chris Callison-Burch. 2015. PPDB 2.0: Better paraphrase ranking, finegrained entailment relations, word embeddings, and style classification. In Proc. ACL.

Jeffrey Pennington, Richard Socher, and Christopher D Manning. 2014. GloVe: Global vectors for word representation. In Proc. EMNLP.

Lorien Y Pratt, Jack Mostow, Candace A Kamm, and Ace A Kamm. 1991. Direct transfer of learned information among neural networks. In Proc. AAAI.

Richard Socher, Alex Perelygin, Jean Y Wu, Jason Chuang, Christopher D Manning, Andrew Y Ng, and Christopher Potts. 2013. Recursive deep models for semantic compositionality over a sentiment treebank. In Proc. EMNLP.

Nitish Srivastava, Geoffrey Hinton, Alex Krizhevsky, Ilya Sutskever, and Ruslan Salakhutdinov. 2014. Dropout: A simple way to prevent neural networks from overfitting. JMLR.

Johan van Benthem. 2008. A brief history of natural logic. In M. Chakraborty, B. Löwe, M. Nath Mitra, and S. Sarukki, editors, Logic, Navya-Nyaya and Applications: Homage to Bimal Matilal. College Publications.

Sida I. Wang and Christopher D. Manning. 2012. Baselines and bigrams: Simple, good sentiment and topic classification. In Proc. ACL.

Rui Wang and Günter Neumann. 2007. Recognizing textual entailment using sentence similarity based on dependency tree skeletons. In ACL-PASCAL Workshop on Textual Entailment and Paraphrasing.

Gregory Ward and Betty Birner. 2004. Information structure and non-canonical syntax. In Laurence R. Horn and Gregory Ward, editors, Handbook of Pragmatics, pages 153-174. Blackwell, Oxford. 
Jason Weston, Antoine Bordes, Sumit Chopra, and Tomas Mikolov. 2015a. Towards AI-complete question answering: A set of prerequisite toy tasks. arXiv:1502.05698.

Jason Weston, Sumit Chopra, and Antoine Bordes. 2015b. Memory networks. In Proc. ICLR.

Terry Winograd. 1972. Understanding natural language. Cognitive Psychology, 3(1):1-191.

Peter Young, Alice Lai, Micah Hodosh, and Julia Hockenmaier. 2014. From image descriptions to visual denotations: New similarity metrics for semantic inference over event descriptions. TACL, 2:6778.

Matthew D. Zeiler. 2012. ADADELTA: an adaptive learning rate method. arXiv preprint arXiv:1212.5701. 